\title{
The study on the potential of muon measurements on the determination of the cosmic ray composition using a new fast simulation technique
}

\author{
Raul R. Prado ${ }^{a}$, Ruben Conceição ${ }^{b}$, Mário Pimenta $^{* b}$, Vitor de Souza ${ }^{a}$ \\ ${ }^{a}$ Instituto de Física de São Carlos - USP, São Carlos, Brazil \\ ${ }^{b}$ Laboratório de Instrumentação e Física Experimental de Partículas, Lisbon, Portugal \\ E-mail: raul.prado@usp.br
}

\begin{abstract}
In this work we study the energy evolution of the number of muons in air showers. Motivated by future plans for UHECR experiments, the analysis developed here focuses on how the evolution of the moments of the distributions of two shower observables, the depth of the shower maximum $X_{\max }$ and the number of muons on the ground $\left(N_{\mu}\right)$, can be used to assess the validity of a mass composition scenario, surpassing the current uncertainties on the shower description. The cosmic ray composition is an essential ingredient for an astrophysical interpretation of the data. However, the inference of composition from air shower measurements is limited by the theoretical uncertainties on the high energy hadronic interactions. Statistical analyses using the energy evolution of different observables, like the moments of the $X_{\max }$ and of the moments of the $N_{\mu}$ distributions, can provide an efficient method to overcome these limitations providing more reliable information about the cosmic ray abundance. A new technique is presented here to generate a large set of simulated shower observables minimizing computer processing time. Fast algorithms to simulate the longitudinal development of the shower (i.e. CONEX) have longbeen available. However, the $N_{\mu}$ is measured along the lateral development of the shower, which implies that three-dimensional simulations are needed (i.e. CORSIKA). This paper presents a parameterization of the main shower characteristics that can be used to simulate the number of muons on the ground using fast simulation algorithms. The parametrization was used to predict the evolution of $\log _{10} N_{\mu}$ moments by possible hypothetic mass composition scenarios.
\end{abstract}

The 34th International Cosmic Ray Conference,

30 July- 6 August, 2015

The Hague, The Netherlands

\footnotetext{
*Speaker.
} 


\section{Introduction}

The determination of the nature of Ultra High Energy Cosmic Rays (UHECRs) is presently one of the biggest challenges of this field. The main difficulty arises from the fact that the primary composition, which is expected to be anything between proton and iron, is determined indirectly through the characteristics of the shower that was induced by the interaction of the primary particle with the nuclei of the atoms in the atmosphere. The measured shower observables, for instance the depth at which the shower reaches the maximum number of particles $\left(X_{\max }\right)$ or the number of muons that reach the ground $\left(N_{\mu}\right)$, are then compared to the predictions from simulation in order to infer the primary composition.

However, these simulations rely on the description of the interactions that occur during the shower development, in particular high-energy hadronic interactions. The latter are expected to carry large uncertainties as there is no first-principles theory to describe these interactions nor available accelerator data at these energies and kinematic regimes to constrain phenomenological models.

Hence, the ability of these simulations to predict the absolute value of the shower observables for a given primary depends on our knowledge about the shower description. Moreover, systematic uncertainties on the energy reconstruction can affect the determination of the primary mass composition at the given energy.

One way to overcome these uncertainties is to look to the evolution of the shower observables with energy instead of analysing only its absolute values. This kind of differential analysis would be, by construction, independent of constant shifts on the energy or on the absolute scale of the shower observables.

Naturally, these kind of studies imply in performing a huge number of simulations over a wide range of energies. While this is not a problem for the $X_{\max }$, which can be simulated with fast 1D hybrid codes, for important observables such as the number of muons on the ground, which have to be simulated with full Monte Carlo, this can be computationally very demanding. Although there are hybrid codes that try to create shortcuts, mainly by the introduction of cascade equations at some steps of the shower development, the resulting computation times and storage size are still very challenging for a full exploitation of this kind of differential analysis.

In this paper we present a novel method that allows us to generate a large number of simulations for the number of muons on the ground. This is done by building a parameterization that relates the number of muons obtained with a fast $1 \mathrm{D}$ code with the one experimentally expected on the ground by a full shower simulation.

To show the power of such a method we consider two possible mass composition scenarios and present the predictions for the evolution of the moments of the distributions of the number of muons on the ground. Finally, we draw conclusions about the obtained results.

\section{Method to build $N_{\mu}$ distributions}

In this section we describe the method used to generate the $N_{\mu}$ distributions for a given primary. The main idea is to convert the total number of muons in the ground with energy above $1 \mathrm{GeV}$ given by CONEX [1] showers $\left(N_{\mu}^{C X}\right)$ to the number of muons which is experimentally observed $\left(N_{\mu}^{\exp }\right)$. 
The conversion algorithm is created using CORSIKA [2] showers to parameterize the relations between $N_{\mu}^{C X}$ and $N_{\mu}^{e x p}$. CONEX is a hybrid simulation code which combines full Monte Carlo, for high-energy particles, with solutions of one-dimensional cascade equations to describe the showers. This makes it much less computationally costly than CORSIKA, and therefore, the parametrization proposed here becomes an efficient method to generate a large set of simulated $N_{\mu}$ data.

$N_{\mu}^{e x p}$ is defined here as the number of muons with energy above $0.2 \mathrm{GeV}$ that reach the ground at a distance between $500 \mathrm{~m}$ and $2000 \mathrm{~m}$ from the shower core. This choice is motivated by the design of the current cosmic ray experiments, like the Pierre Auger Observatory [3] and the Telescope Array [4]. The limited axis distance comes from the granularity of the detectors arrays, which does not allow the measurement of the full lateral distributions of particles, and the energy limit comes from the usual threshold of scintillators and water-Cherenkov detectors.

The conversion of $N_{\mu}^{C X}$ into $N_{\mu}^{e x p}$ is done through the following relation: $N_{\mu}^{e x p}=R\left(E, X_{\max }\right)$. $N_{\mu}^{C X}$, where $R$ is a quantity that should be determined for each primary and depends on energy and $X_{\max }$. Figure 1 shows the factor $R$ obtained from CORSIKA showers as a function of $X_{\max }$ for proton initiated showers at $E=10^{19} \mathrm{eV}$ and for EPOS-LHC [5]/FLUKA [6] as high/low energy hadronic interaction models.

The behavior of $R\left(X_{\max }\right)$ allows us to perform a linear parameterization in the form $R\left(X_{\max }\right)=$ $p_{1} \cdot X_{\max }+p_{0}$. Afterwards, $p_{1}$ and $p_{0}$ are parameterized as a function of energy for each primary, as shown in figure 2. This process was performed by using a set of 200 CORSIKA showers for each fixed energy $\left(10^{18.5}, 10^{19.0}\right.$ and $10^{19.5} \mathrm{eV}$ ) and each primary (proton, helium, nitrogen and iron). The zenith angle was set to $38^{\circ}$. Naturally, the choice of a different high-energy hadronic interaction models implies different parameters.

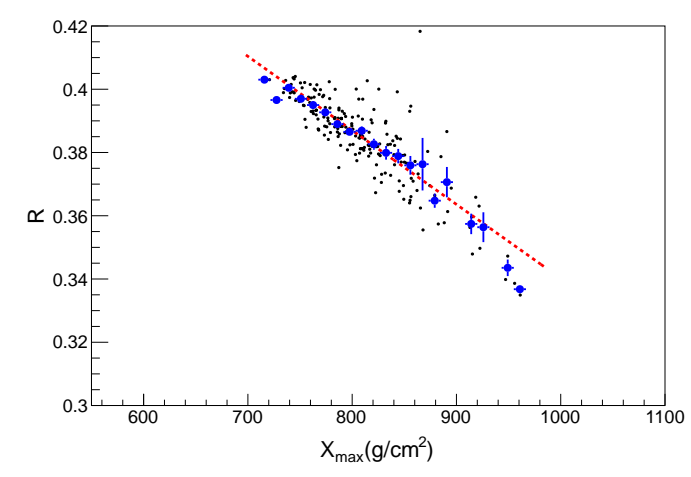

(a)

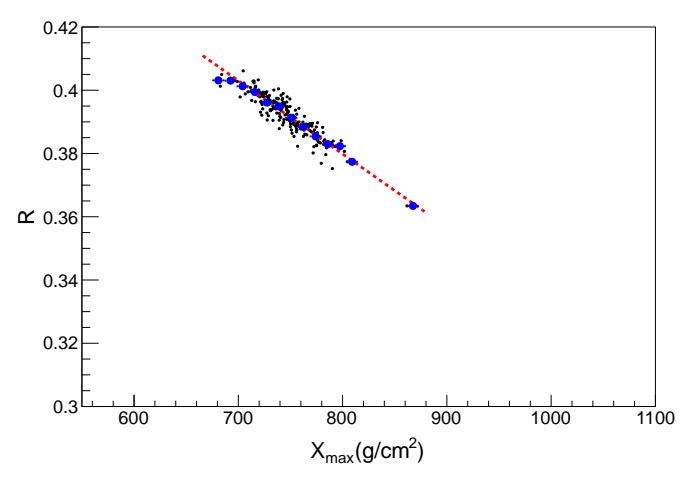

(b)

Figure 1: Conversion factor $R$ obtained from 200 CORSIKA showers initiated by protons (left) and nitrogen (right) at $10^{19.0} \mathrm{eV}$. Black dots represent individual showers, blue dots the profile and the red dashed lines are the linear fit. EPOS-LHC [5] and Fluka [6] were used as high and low energy hadronic interaction models respectively.

The $N_{\mu}^{e x p}$ distributions obtained through the method developed in this work are shown in figure 3. In this figure the $N_{\mu}$ distribution from CORSIKA showers generated with the same energy, $E=10^{19} \mathrm{eV}$, are also shown for comparison. The moments of these distributions are in agreement within $2 \%$ for the average and $5 \%$ for the variance. 


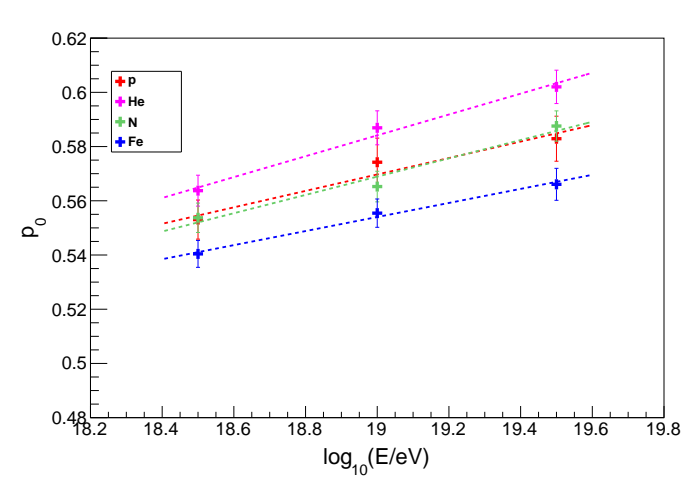

(a)

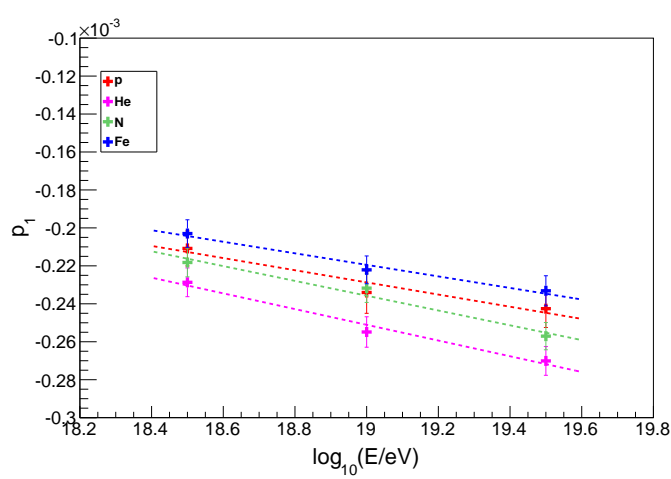

(b)

Figure 2: $p_{0}$ (left) and $p_{1}$ (right) parameterization for EPOS-LHC. These parameters describe the relation of the conversion factor $R$ and $X_{\max }$, given by $R\left(X_{\max }\right)=p_{0} \cdot X_{\max }+p_{1}$.

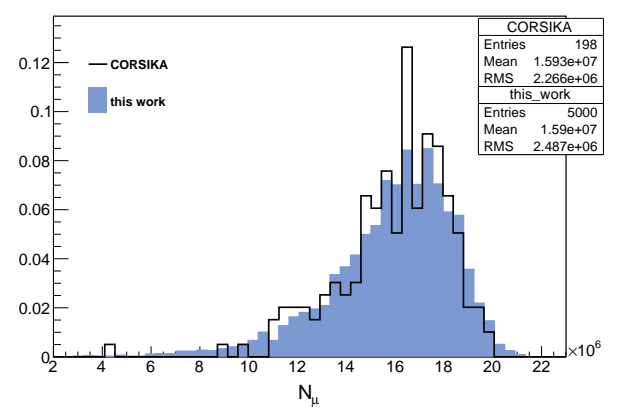

(a) Proton

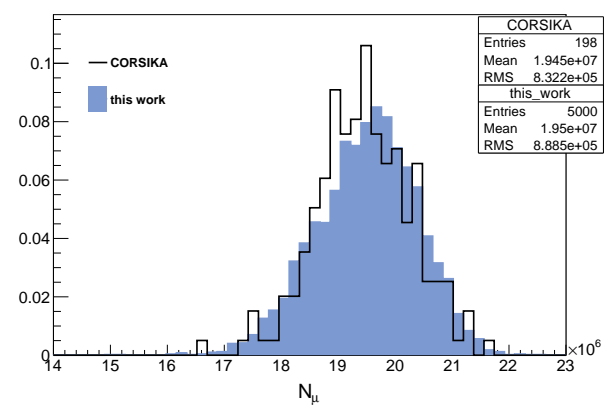

(c) Nitrogen

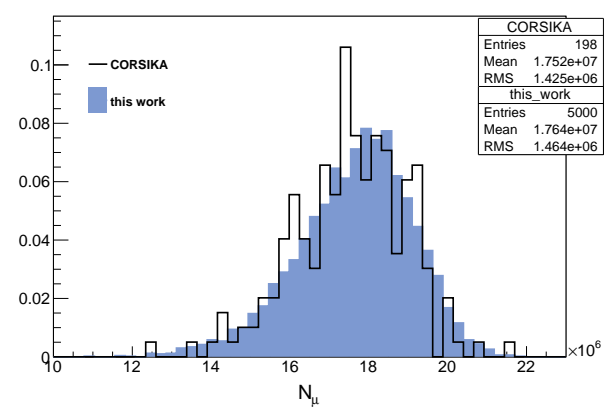

(b) Helium

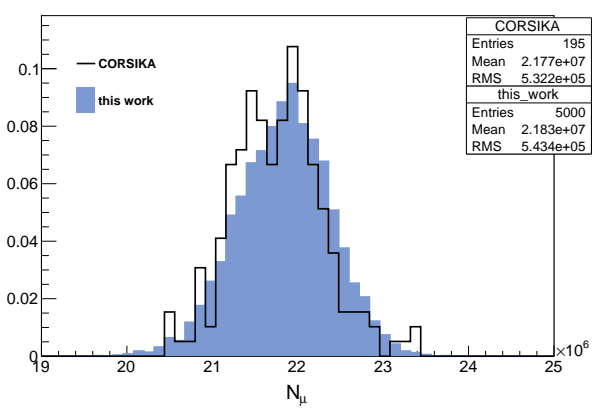

(d) Iron

Figure 3: Comparison between $N_{\mu}$ distributions obtained by this method and from CORSIKA showers. The hadronic interaction models are EPOS-LHC/FLUKA and the primary energy is $E=10^{19.0} \mathrm{eV}$.

\section{Mass Composition Scenarios}

In order to test the potentiality of muon measurements in discriminating the cosmic ray composition, we have chosen two possible scenarios of mass composition evolution. One is inspired by the composition fits performed to the Pierre Auger Observatory $X_{\max }$ measurements and another 
comes essentially from astrophysical considerations. These scenarios are described below.

- Auger $X_{\max }$ : It was shown by Pierre Auger Collaboration that the measured $X_{\max }$ distributions can be well described by a mixture of four components (proton, helium, nitrogen and iron) [7]. The fractions of each component were obtained by fitting to the data a set of Monte Carlo simulations generated by the two recently updated hadronic interaction models (QGSJetII-04 [8] and EPOS-LHC [5]). One of the composition scenarios used in this work is based on the results of the $X_{\max }$ distributions fits for EPOS-LHC.

- Allard et al.: The second example of composition scenario was proposed by Allard et al.[9] and has astrophysical motivations. In this model it is assumed that the sources of UHECR are extragalactic with mixed composition. The ankle in the spectrum is well described, as well as the $X_{\max }$ measurements up to the date of the publication of the scenario. In Ref. [9] this model is called Model A.

The fractions for each component as a function of primary energy for each scenario are shown in figures $4 \mathrm{a}$ and $4 \mathrm{~b}$. A polynomial fit was performed in the Auger $X_{\max }$ scenario in order to make the energy evolution of each component smooth. Besides these two scenarios, the pure proton and pure iron compositions are also shown as reference.

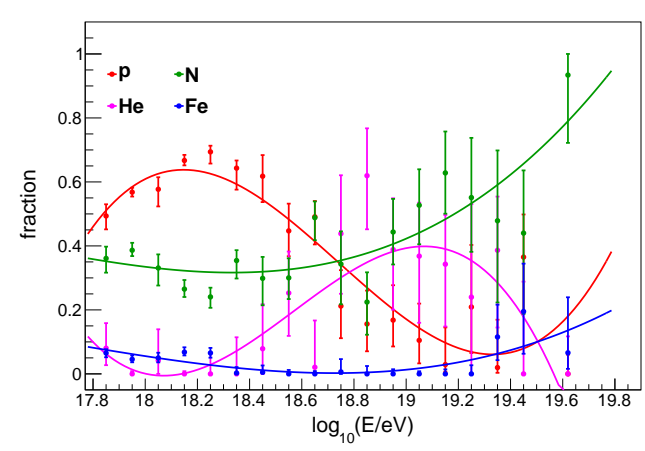

(a) Auger $X_{\max }[7]$

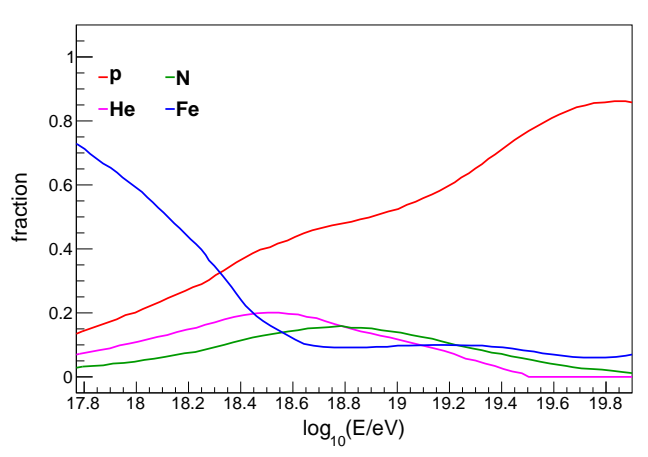

(b) Allard et al. [9]

Figure 4: Fractions of the composition components as a function of energy for each composition scenario. See the text for details.

\section{Energy Evolution of $\log _{10} N_{\mu}$ Moments}

Using the method developed to produce the distributions of the number of muons on the ground we can now investigate the expected energy evolution of $\log N_{\mu}$, its first and second moment, for the composition scenarios introduced in the previous section. The CONEX simulation set used in this analysis contains 20000 showers in each energy bin of $\Delta \log _{10} E=0.1$ for each primary (proton, helium, nitrogen and iron), uniformly distributed in $\log _{10} E$. The zenith angle was chosen to be fixed at $38^{\circ}$. The contribution of each shower to the distributions of $\log _{10} N_{\mu}$ is weighted according to the cosmic ray energy spectrum measured by Pierre Auger Observatory [10] and the primary fraction in a given composition scenario. 


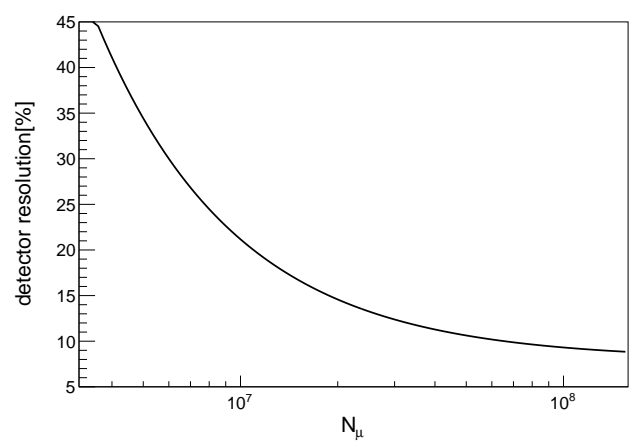

Figure 5: Detector resolution as a function of $N_{\mu}$ for both cases considered in this note. The plot shows the relative resolution, defined as $100 \times \frac{\sigma_{N_{\mu}}}{N_{\mu}}$, where $\sigma_{N_{\mu}}$ is the width of the Gaussian function used to smear the $N_{\mu}$ distribution.

In order to get a more realistic assessment of the evolution of the observables with energy the detector resolution was also taken into account. This was emulated by applying a Gaussian smearing of width $\sigma_{N_{\mu}}$ to the $N_{\mu}$ distributions. In figure 5 we show the detector resolution considered in this work. The resolution is $30 \%$ for $N_{\mu}=6 \cdot 10^{6}\left(E \sim 10^{18.5} \mathrm{eV}\right.$ for proton induced showers) and the asymptotic detector resolution for high energies is $8 \%$.

In figures $6 \mathrm{a}$ and $6 \mathrm{~b}$ we show the evolution of $\left\langle\log _{10} N_{\mu}\right\rangle$ and $R M S\left(\log _{10} N_{\mu}\right)$ with energy without taking into account the detector resolution. The plotted values are divided by the proton (iron) expectation values for $\left\langle\log _{10} N_{\mu}\right\rangle\left(R M S\left(\log _{10} N_{\mu}\right)\right)$ for better visualization. The error bars represent the expected statistical uncertainty for 3 years of data taking with the full Pierre Auger Observatory array and events with a zenith angle within $\theta \in\left[20^{\circ} ; 40^{\circ}\right]$. In figures $6 \mathrm{c}$ and $6 \mathrm{~d}$ we show the same plots but taking into account the detector resolution presented in figure 5 .

One can clearly see from the plots on figure 6 that predicted composition scenarios are significantly different concerning the energy evolution of the $\log _{10} N_{\mu}$ moments.

Even if the comparison with data were to be hampered due to uncertainties on the hadronic interaction models or the absolute energy scale, the trending of the scenarios could help to verify/falsify them. This is extremely important as we have indications that the Monte Carlo simulations present a deficit with respect to data [11], and therefore the absolute values are not reliable.

Furthermore, the differences as predicted from simulations for both composition scenarios can suggests further studies on the possibility to distinguishing among composition scenarios by quantifying structures in the energy evolution of the $\log _{10} N_{\mu}$ moments.

\section{Conclusions}

In this paper we presented a fast simulation technique which enables the generation of a large set of simulated showers to be used for performing statistical analysis of $N_{\mu}$ distributions. This simulation method was used in order to predict the energy evolution of $\log _{10} N_{\mu}$ first and second moments for two possible mass composition scenarios.

With this exercise it has been shown qualitatively that the mass composition scenarios can be distinguished not only on the absolute value but also assessing the energy evolution of $\log _{10} N_{\mu}$ 


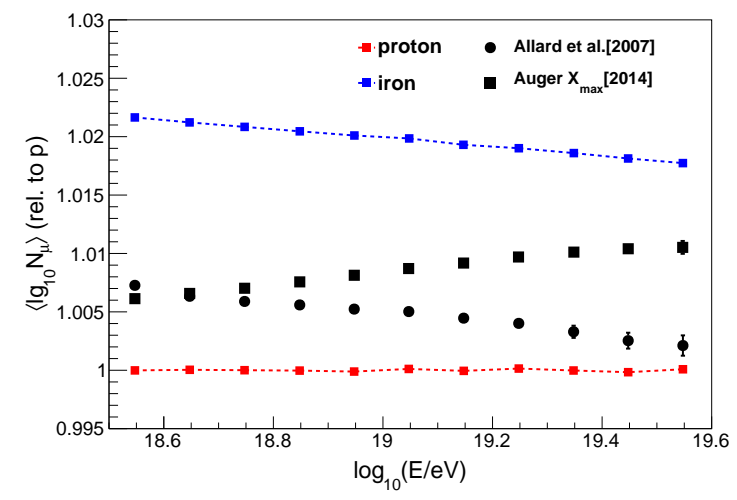

(a)

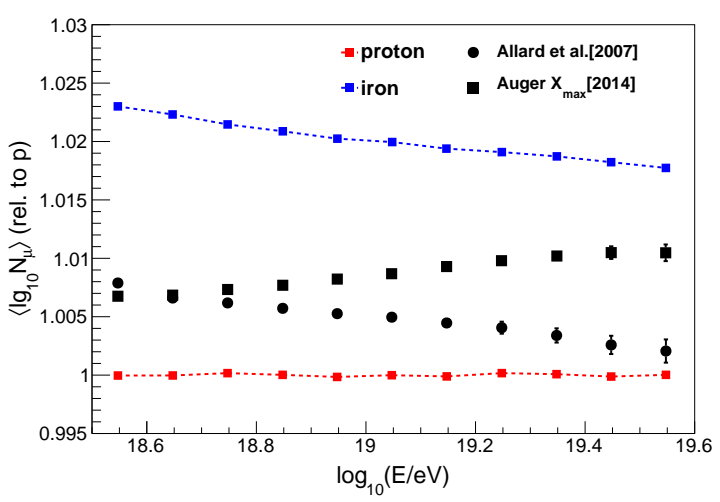

(c)

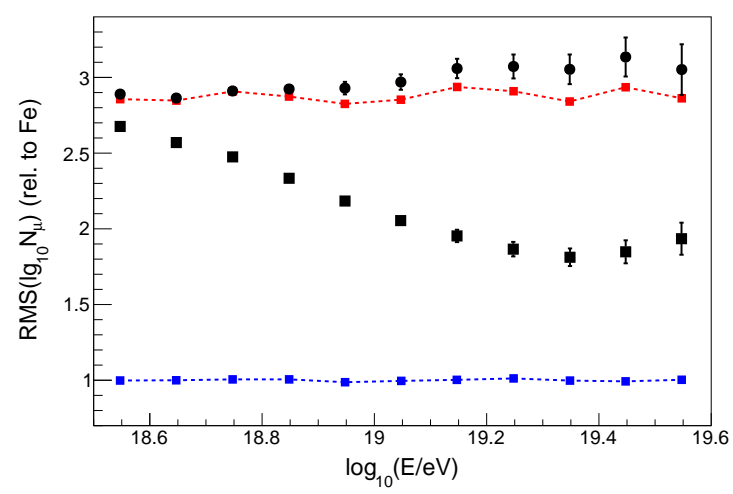

(b)

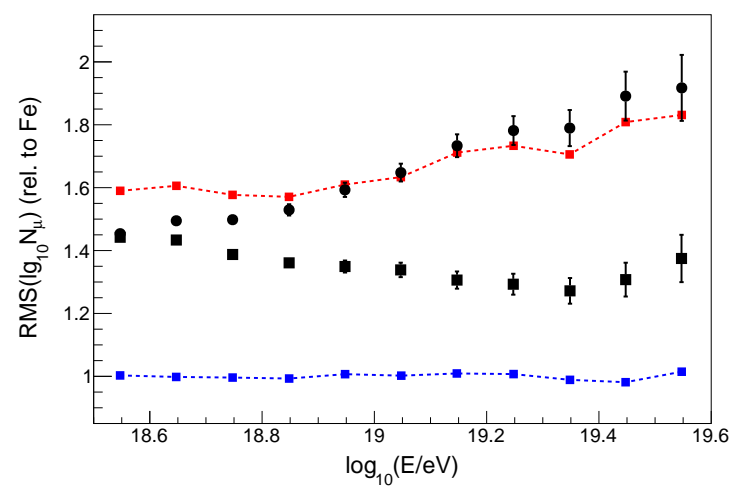

(d)

Figure 6: Energy evolution of $\left\langle\log _{10} N_{\mu}\right\rangle$ and $R M S\left(\log _{10} N_{\mu}\right)$. The values are divided by proton values (left column) for $\left\langle\log _{10} N_{\mu}\right\rangle$ and by iron values (right collumn) for $R M S\left(\log _{10} N_{\mu}\right)$. In (a) and (b) no detector resolution is included, and in (c) and (d) the detector resolution is considered.

moments. The latter is of the utmost importance as it is insensitive to shifts on the absolute scale of the observables caused by the uncertainties on the hadronic interactions or on the absolute energy reconstruction.

\section{Acknowledgments}

R. R. P. and V. S. acknowledge the financial supports from FAPESP (2010/07359-6 and 2014/10460-1) and CNPq. R. C. acknowledges the financial support of Fundação para a Ciência e Tecnologia (SFRH/BPD/73270/2010).

\section{References}

[1] T. Bergmann et al. One-dimensional hybrid approach to extensive air shower simulation. Astropart. Phys., 26, 6 (2007).

[2] D. Heck et al. CORSIKA: A Monte Carlo code to simulate extensive air showers. FZKA Report, 6019, 1998. 
[3] A. Aab et al. [Pierre Auger Collaboration] The Pierre Auger Cosmic Ray Observatory, accepted for publication in Nucl. Instrum. Meth. A (2015). [arXiv:1502.01323]

[4] H. Kawai et al. [Telescope Array Collaboration], Telescope Array Experiment, Nucl. Phys. B Proc. Sup., 175-176, 221 (2008).

[5] K. Werner and T. Pierog, Extended Air Shower Simulations Using EPOS, In AIP Conference Proceedings, 928 (2007) 111-117. AIP. [arXiv:0707.3330].

[6] G. Battistoni et al. The FLUKA code: Description and benchmarking, In AIP Conference Proceedings, 89631 (2007).

[7] A. Aab et al. [Pierre Auger Collaboration], Depth of maximum of air-shower profiles at the Pierre Auger Observatory. II. Composition implications, Phys. Rev. D 90, 122006 (2014). [arXiv:1409.4809]

[8] S. Ostapchenko, Monte Carlo treatment of hadronic interactions in enhanced Pomeron scheme: I. QGSJET-II model, Phys. Rev. D 83, 014018 (2011). [arXiv:1010.1869]

[9] D. Allard, E. Parizot and A. V. Olinto, On the transition from galactic to extragalactic cosmic-rays: Spectral and composition features from two opposite scenarios, Astropart. Phys. 27, 61 (2007). [arXiv:astro-ph/0512345]

[10] A. Schulz. [Pierre Auger Collaboration], The measurement of the energy spectrum of cosmic rays above $3 \times 10^{17} \mathrm{eV}$ with Pierre Auger Observatory, Proc. of 33rd ICRC(2013) [arXiv:1307.5059]

[11] A. Aab et al. [Pierre Auger Collaboration], Muons in air showers at the Pierre Auger Observatory: Mean number in highly inclined events, Phys. Rev. D 91, 032003 (2015). [arXiv:1408.1421] 